EPJ Web of Conferences 102, 00001 (2015)

DOI: $10.1051 /$ epjconf/201510200001

(C) Owned by the authors, published by EDP Sciences, 2015

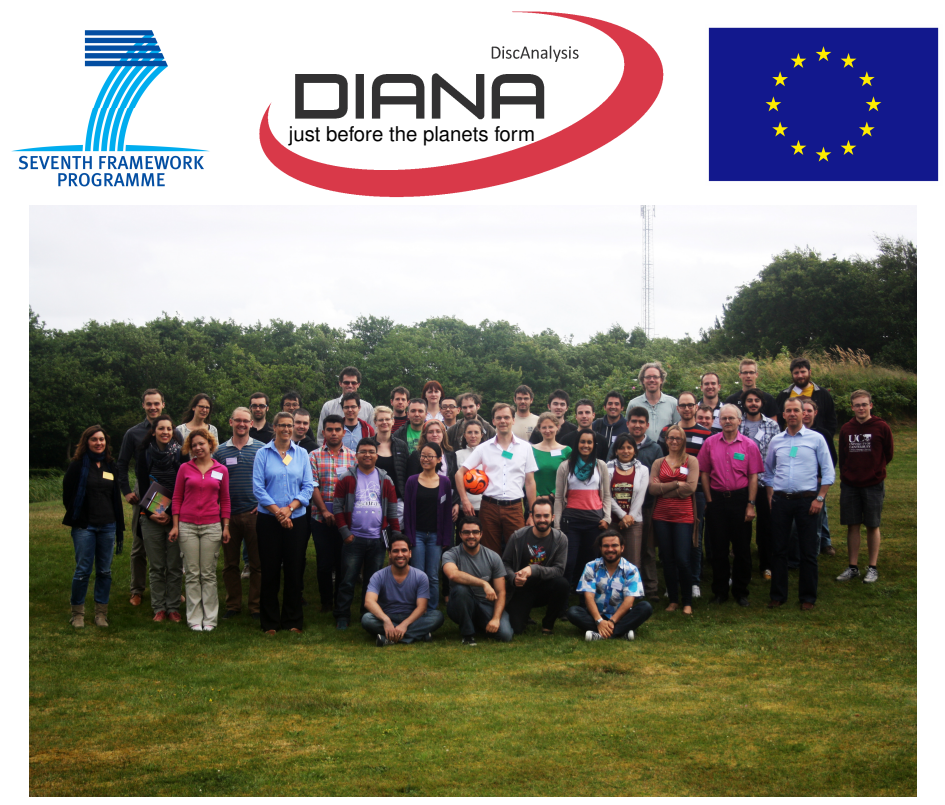

\title{
Preface
}

The formation of protoplanetary disks and planets around new-born stars closely relates to the fundamental question "Are we alone in the universe?". Hence, the study of disks and their link to planet formation is undoubtedly one of the main science drivers for current astrophysical research worldwide, and for the design of future telescopes and astronomical instrumentation. The observational study of protoplanetary disks, the birthplaces of exoplanets, requires a carefully concerted, multiwavelength and multi-instrument approach to reveal the physical, thermal, and chemical structure of dust and gas in different parts of the disks.

The FP7 project DiscAnalysis (DIANA) has established a major European initiative to collect public and proprietary multi-wavelength continuum and line data for protoplanetary disks, and analyse and interpret these data by means of novel high-quality disk models, see http://www.dianaproject.com/. In the summer school Protoplanetary Disks: Theory and Modeling meet Observations, we discuss the available types of observational data, and introduce the theoretical concepts and foundations that are built into our current radiative transfer and thermo-chemical models.

The summer school was held from 16th to the 20th of June 2014 at the Hotel Amelander Kaap on the Island of Ameland, The Netherlands. The School was attended by $45 \mathrm{PhD}$ students and post-docs from 19 countries around the world. Ten lecturers from the DIANA team explained basic theories ranging from the formation and evolution of protoplanetary disks, chemistry and radiative transfer in disks to the diversity of observational data such as SEDs, images, line emission and interferometry. Several lectures focused on disk modeling and its application to observations including limitations, pitfalls and outlook to new instrumentation. We hope that this collection of lectures contributes to the development of new university lecture courses about star and planet formation, and that it will inspire the next generation of scientists to continue unraveling the mysteries involved.

Inga Kamp, Peter Woitke, John Ilee

This is an Open Access article distributed under the terms of the Creative Commons Attribution License 4.0, which permits unrestricted use, distribution, and reproduction in any medium, provided the original work is properly cited. 


\section{List of Lecturers}

Dionatos, Odysseas

Dominik, Carsten

Güdel, Manuel

Greaves, Jane

Kamp, Inga

Min, Michiel

Pinte, Christophe

Thi, Wing-Fai

Waters, Rens

Woitke, Peter
Department of Astrophysics, University of Vienna, Austria

Anton Pannekoek Institute, Amsterdam, The Netherlands

Department of Astrophysics, University of Vienna, Austria

School of Physics \& Astronomy, University of St Andrews, Scotland

Kapteyn Astronomical Institute, University of Groningen, The Netherlands

Anton Pannekoek Institute, Amsterdam, The Netherlands

UMI-FCA, Santiago, Chile

Institut de Planétologie et d'Astrophysique de Grenoble, France

SRON, Utrecht, The Netherlands

School of Physics \& Astronomy, University of St Andrews, Scotland 


\section{List of Participants}

Antonellini,

Baldovin Saavedra,

Beiranvand,

Bewketu,

Caceres,

Carmona,

Christiaens,

Csepany,

D'Angelo,

de Juan Ovelar,

Demidova,

Dionatos,

Dipierro,

Dominik,

Elbakyan,

Faes,

Georgetti Vieira,

Greenwood,

Güdel,

Hardy,

Ilee,

Greaves,

Kama,

Kamp,

Liu,

Maucó,

Min,

Montesinos,

Muthusubramanian,

Navarete,

Pearson III,

Perez,

Pinilla,

Pinte,

Ramsey,

Rendón Acosta,

Ribas,

Rigon,

Robson Monteiro Rocha,

Rodgers-Lee,

Rosales Guzmán,

Sahin,

Salinas,

Steiner,

Stolker,

Szegedi-Elek,

Teague,

Thi,

Tocknell,

Traven,

Ulbrich,

Vicente,

Waters,

Woitke,

$\mathrm{Wu}$,

$\mathrm{Yu}$,

Zakhozhay,
Stefano

Carla

Nassim

Asnakew

Claudio

Andres

Valentin

Gergely

Martina

Maria

Tatiana

Odysseas

Giovanni

Carsten

Vardan

Daniel M.

Rodrigo

Aaron

Manuel

Adam

John

Jane

Mihkel

Inga

Chun-Fan

Karina

Michiel

Matias

Balaji

Felipe

Richard

Sebastian

Paola

Christophe

Jon

Francisco

Álvaro

Laura

Will

Donna

Jaime Andrés

Timur

Vachail

Daniel

Tomas

Elza

Richard

Wing-Fai

James

Gregor

Kathrin

Silvia

Rens

Peter

Chao-Jian

Mo

Olga
Kapteyn Astronomical Institute, University of Groningen, The Netherlands Department of Astrophysics, University of Vienna, Austria

Tehran, Iran

Madawalabu University, Ethiopia

Universidad de Valparaiso, Chile

Institute de Planetologie et Astrophysique de Grenoble, France

University of Chile, Chile

ESO, Garching bei München, Germany

University of Groningen, The Netherlands

Astrophysics Research Institute, Liverpool, John Moores University, UK

University of Saint-Petersburg, Russia

Department of Astrophysics, University of Vienna, Austria

Milano, Italy

Anton Pannekoek Institute, Amsterdam, The Netherlands

Rostov-on-Don, Russia

IAG-USP, Sao Paulo, Brazil

Universidade de Sao Paulo, Brazil

Kapteyn Institute, University of Groningen, The Netherlands

Department of Astrophysics, University of Vienna, Austria

Universidad de Valparaiso Faculdad de Ciencias, Chile

School of Physics \& Astronomy, University of St Andrews, Scotland

School of Physics \& Astronomy, University of St Andrews, Scotland

Leiden Observatory, The Netherlands

Kapteyn Astronomical Institute, University of Groningen, The Netherlands

Graduate Institute of Astrophysics, National Taiwan University, Taiwan

Michoacán, Mexico

Anton Pannekoek Institute, Amsterdam, The Netherlands

Pontificia Universidad Catolica de Chile, Santiago, Chile

Kapteyn Institute of Astronomy, University of Groningen, The Netherlands

Cidade Universitária, São Paulo, Brazil

University of Denver, USA

Universidad de Chile, Santiago, Chile

Leiden Observatory, The Netherlands

UMI-FCA, Santiago, Chile

Institut für Theoretische Astrophysik, Heidelberg Universität, Germany

Departamento de Astronomía de la Universidad de Guanajuato, Mexico

European Space Astronomy Centre, Madrid, Spain

School of Physics \& Astronomy, University of St Andrews, Scotland

UNIVAP, São Paulo, Brazil

Dublin Institute for Advanced Studies, School of Cosmic Physics, Ireland

Concepción, Chile

Akdeniz University, Merkez, Antalya, Turkey

Leiden Observatory, The Netherlands

Department of Astrophysics, University of Vienna, Austria

Anton Pannekoek Institute, Amsterdam, The Netherlands

Budapest, Hungary

Max-Planck-Institut für Astronomie, Heidelberg, Germany

Institut de Planétologie et d'Astrophysique de Grenoble, France

Macquarie University, Sydney, Australia

University of Ljubljana, Slovenia

Institut für Astrophysik, Göttingen, Germany

Kapteyn Astronomical Institute, University of Groningen, The Netherlands SRON, Utrecht, The Netherlands

School of Physics \& Astronomy, University of St Andrews, Scotland

National Astronomical Observatories, Chinese Academy of Sciences, China

The University of Texas at Austin, Department of Astronomy, USA

Kyiv, Ukraine 